\title{
Technological properties of potato starch treated by Heat-Moisture Treatment with addition of organic acids
}

\author{
Jéssica Iwasenko Giacomozzi ${ }^{1}$, Bárbara Ruivo Válio Barretti ${ }^{2}$, \\ Vanessa Soltes de Almeida ${ }^{1}$, Camila Delinski Bet ${ }^{1}$, \\ Marco Aurélio da Silva Carvalho Filho ${ }^{3,4}$, Luiz Gustavo Lacerda ${ }^{1}$, \\ Ivo Mottin Demiate ${ }^{1}$, Egon Schnitzler ${ }^{1}$
}

\author{
1 - State University of Ponta Grossa, Brazil \\ 2 - Federal University of Parana, Brazil \\ 3 - Pontifical Catholic University of Paraná, Brazil \\ 4 - Posivito University, Brazil
}

\section{Keywords:}

Starch

Modification

HMT

Lactic acid

Citric acid

Article history:

Received 20.09.2020

Received in revised

form 10.12.2020

Accepted 25.03.2021

Corresponding

author:

Egon Schnitzler

E-mail:

egons@uepg.br

DOI: $10.24263 / 2304-$

974X-2021-10-1-8

\section{Abstract}

Introduction. Starch has been modified to overcome industrial limitations present in its native form. Heat-moisture treatment (HMT) is essentially a physical and thermal method applied to modify starch. It can be combined with some chemicals to provide unique technological characteristics to these biopolymers.

Materials and methods. In this study, the potato starch was modified by conventional HMT using water and with $0.2 \mathrm{~mol} \mathrm{~L}^{-1}$ solutions of the organic acids lactic and citric up to $22 \%$ ratio. The influence on pasting and thermal properties of the potato starch was investigated. With the action of HMT, with water and acid, it was possible to observe structural changes in the starch.

Results and discussion. The commercial potato starch shown BC pattern type (B type with characteristics of $C$ type). The treated starches have a $\mathrm{C}$ pattern type, with main diffraction peaks $2 \theta$ at $5.6^{\circ}, 15.3^{\circ}, 17.3^{\circ}$ and $23.5^{\circ}$, respectively. A decrease in intensity peaks at $5.6^{\circ}$ according each treatment was observed. The relative crystallinity (RC) of each starch was calculated being $27.2 \%$ from commercial potato starch which decrease to $19.9 \%$ to the treated with citric acid. Through pasting properties analysis (RVA) it was possible to observe that the viscosity peak presented a notable reduction from 7,824.00 mPa/s (native), to $90.00 \mathrm{mPa} / \mathrm{s}$ (HMT + citric acid). The Differential Scanning Calorimetry (DSC) was used to determine the values of onset $\left(T_{o}\right)$, peak $\left(T_{p}\right)$ and endset $\left(\mathrm{T}_{\mathrm{c}}\right)$ temperatures of gelatinization as well as the enthalpy $\left(\Delta \mathrm{H}_{\mathrm{gel}}\right)$. The native (commercial) starch + HMT and the one treated with $22 \%$ water + HMT shown enthalpy 14.5 and 9,9 $\mathrm{J} \mathrm{g}^{-1}$, respectively. The others, treated with $22 \%$ solution of lactic acid + HMT and $22 \%$ solution of citric acid + HMT showed an enlargement between $T_{o}$ and $T_{c}$ with drastic reduction of viscosity.

Conclusions. The main application of this modified starch is in foods that require low viscosity, such baby food, soups and as an ingredient for baked products like cookies. 


\section{- Food Technology -}

\section{Introduction}

Starch is a biodegradable polysaccharide, from a renewable source. It is composed mainly by amylose and amylopectin molecules. The amylose in which each unit of glucose is linked by $\alpha-(1 \rightarrow 4)$ glycosidic bonds, and the amylopectin, which molecule is highly branched by glycosidic portions of both $\alpha-(1 \rightarrow 4)$ and $\alpha-(1 \rightarrow 6)$ glycosidic bond (Yassaroh et al., 2019). This biopolymer is the main storage carbohydrate produced by plants. It is widely processed and consumed by human as an energy source (Leonel et al., 2011). The source of starch can be very broad. The most used sources for human consumption regards to starches from cereals and tubers such as corn, wheat, potato and cassava starch (Zaman et al., 2016). The World Health Organization (WHO) recommends that more than $55 \%$ of the energy ingested by humans should be from carbohydrates, which means that studies on starches and their health benefits are highlighted (Pereira,2017). As ingredient starch is responsible for technological properties that characterize most products, contributing with texture, thickeness, colloidal stabilization, gelling, volume, adhesiveness, water retention, and many others (Jiranuntakul et al., 2011).

Potato (Solanum tuberosum L.) is the third most important food crop on the planet; it is the first non-grain commodity (dos Santos et al., 2016). This tuber has a significant importance when we talk about starch, because although most of its production is destined for fresh consumption. Potato starch, has been gaining market with thermal and thickening application in the food industry (Noda et al., 2006). Potato starch is composed of approximately $80 \%$ amylopectin and 20\% amylose. In comparison with cereal starches, potato starch, gel forms with high viscosity, good consistency and clarity. Due to the presence of phosphate, gelatinized material shows high transparency (Noda et al., 2006; Shin et al., 2007). All these characteristics make it interesting to be used as thickeners for dehydrated soups and sauces, binding agents in sausages, puddings and desserts (dos Santos et al., 2016).

Despite having value in the industry, use of native or untreated starch present several problems such as handling, tendency to retrogradate, low freeze-thaw stability, and poor tolerance to many technological processes such as poor thermal resistance and low shear resistance (Barreti et al., 2020). To improve this limitation, starch granules can be modified by several methods, such as chemical, physical, biological and even combined processes (Schafranski et al., 2021). Among the most used starch modification methods in the industry, the most well-known are heat-moisture, dry heat, annealing, pre-gelatinization, high pressure, radiation, ultrasound, cross-linking, substitution, acid hydrolysis and oxidation treatments (Noda et al., 2006). The starch modification extent is affected by botanical source, composition, proportion of amylose to amylopectin, as well as by the disposition of the chains within the amorphous and crystalline regions of the starch granules (Schafranski et al., 2021).

HMT is essentially a physical modification comprises of heat-treating starch with low moisture content (10-30\%), and high temperatures above starch gelatinisation $\left(90-120{ }^{\circ} \mathrm{C}\right)$. This type of method modifies the physicochemical properties of starch without destroying its granular structure. The first research to use HMT to modify a cereal starch was reported in 1944, and since then several studies have been carried out to investigate the effects of this method applied to different botanical sources. Besides other modification methods like chemical and biological, HMT brings high effectiveness, simplicity, low costs and mainly the non-generation of hazardous chemicals residues. Thus, HMT can be an attractive technique for industrial food applications (Zavareze et al., 2011). Recently, some published studies have shown that the addition of organic acids solutions instead or combined with water (moisture) during HMT process is able to modify starch, due to partial hydrolysis and promoting structural alterations. These researches have been carried out using lactic, citric, and acetic acids at lower concentrations with the advantage of being food grade ingredients. Furthermore, they can be 
used without limitations when inorganic acids are added during food applications (Barreti et al., 2020). Thus, the granules have been modified to be suitable for further applications. Modified starch is responsible for the main technological properties that configure many processed products.

Technological aspects and impacts provided by starch modifications can be observed by several analytical methods such as differential scanning calorimetry (DSC), powder x-ray diffractometry (PXRD), rapid visco analyser (RVA), among many others. DSC provides information regarding starch gelatinization, which is a regular event during an industrial process. DSC can provide the difference in enthalpy between a sample and a reference (normally an empty pan) as a function of temperature or time under controlled heating and cooling program. Furthermore, the equipment helps to obtain the event beginning or onset temperatures (To), endothermic peak (Tp), conclusion temperature (Tc), and gelatinisation enthalpy difference $(\Delta \mathrm{H})$ (Schafranski et al., 2021). RVA is a rotational viscometer that can measure the shear resistance or pasting properties of a sample. In addition, the equipment can reproduce thermal processing conditions when a sample is heated and cooled. During RVA analysis it is possible to obtain some important information such as maximum viscosity reached during the heating period and tendency to retrograde during cooling process (Barreti et al., 2020). PRXD is a useful tool to evaluate the impacts on the characteristics of starch crystalline structures. It is possible to observe the behaviour of peaks intensities, the pattern characteristic and the relative crystallinity (Zavareze et al., 2011).

Potato starch has been modified to overcome industrial limitations present in its native form, generating more suitable industrial products (Colussi et al., 2020). The modification technique, such as HMT (Heat-moisture treatment) combined with the addition of others, can bring more expressive and interesting results from the functional point of view, results that have been proven in several studies (dos Santos et al., 2016; Hung et al., 2016). Thus the present research aimed to investigate the impact of treatment by HMT using water (moisture) and solutions of lactic acid and citric acid on the structure and the pasting and thermal properties of the potato starch.

\section{Materials and methods}

\section{Samples and reagents}

The potato starch was purchased at a local supermarket in the city of Ponta Grossa $\left(25^{\circ}\right.$ $05^{\prime} 42^{\prime \prime} \mathrm{S}, 5^{\circ} 09^{\prime} 43^{\prime \prime} \mathrm{W}$ ) Paraná, Brazil. The reagents for carrying out the analysis were at least analytical grade.

\section{Methods}

\section{Heat-moisture-treatment (HMT) and Acid HMT}

$30 \mathrm{~g}$ of potato starch (dry base) was suspended in deionized water and in different solutions of citric acid and lactic acid each at $0.2 \mathrm{~mol} \mathrm{~L}^{-1}$. Then, the samples treated in an acid medium were neutralized with sodium hydroxide $0.1 \mathrm{~mol} \mathrm{~L}^{-1}$ and then washed with deionized water. After, the slurry was filtered and dried, obtaining the adjusted level of final humidity at $22 \%$ and placed in hermetically sealed bottles. The samples were so maintained for $24 \mathrm{~h}$ at room temperature and then submitted to $110^{\circ} \mathrm{C}$ in an oven (Tecnal, TE 394/1, Piracicaba, SP, Brazil) for $8 \mathrm{~h}$. Until the analyzes were carried out, the samples were stored in a desiccator with anhydrous calcium chloride (Hung et al., 2016). The treated samples were identified as: native (commercial sample), HMT $\mathrm{H}_{2} \mathrm{O}$ (heat-moisture treatment with 22\% deionized water); HMT LA (heat-moisture treatment with 22\% lactic acid) and HMT CA (heat-moisture treatment with $22 \%$ citric acid). 


\section{Powder X-Ray diffractometry (PXRD)}

Diffractograms of samples were obtained using Ultima IV X-ray diffractometer (Rigaku, Japan) under following conditions: CuK $\alpha$ radiation $(\lambda=1.541 \AA)$, voltage of $40 \mathrm{kV}$ and current of $20 \mathrm{~mA}$. The observed interval was from 3 to $40^{\circ}$ to $2(\theta)$, step of $0.02^{\circ}$ with scanning speed of $2^{\circ} \min ^{-1}$ (Kubiaki, 2018). The main diffraction peaks were observed and Equation (1) was used to calculate the relative crystallinity (Hung et al., 2016; Shaikh et al., 2019):

$$
\mathrm{X}_{\mathrm{c}}=\mathrm{A}_{\mathrm{p}} /\left(\mathrm{A}_{\mathrm{p}}+\mathrm{A}_{\mathrm{b}}\right) 100
$$

where $X_{c}$ refers to relative crystallinity; $A_{p \mid}$ refers to the area of the X-ray diffractogram; and $A_{b}$ refers to the amorphous area of the diffractogram.

\section{Pasting properties (rapid visco analyser - RVA)}

The pasting properties of the samples were determined with RVA-4 instrument (Newport Scientific, Australia). A dispersion in water of $8 \%(\mathrm{~m} / \mathrm{m})$ of starch on a dry basis in $28 \mathrm{~g}$ of total mass, was subjected to a cycle of heating and cooling under constant agitation, where they were kept at $50{ }^{\circ} \mathrm{C}$ for $2 \mathrm{~min}$, heated by 50 to $95^{\circ} \mathrm{C}$ to $6{ }^{\circ} \mathrm{C} \mathrm{min}^{-1}$ and maintained at $95^{\circ} \mathrm{C}$ for $5 \mathrm{~min}$; cooled to $50^{\circ} \mathrm{C}$ to $6{ }^{\circ} \mathrm{C} \mathrm{min}^{-1}$ and held at $50{ }^{\circ} \mathrm{C}$ for $2 \mathrm{~min}$ (Maior at al., 2020).

\section{Differential Scanning Calorimetry (DSC)}

The DSC curves were obtained in a DSC 60 equipment (Shimadzu, Japan). The mass of the starch samples $(2.5 \mathrm{mg})$ were weighed aluminum crucibles and it was added water up to a ratio 1:4 starch water. The crucibles were sealed, maintained by $60 \mathrm{~min}$ and so performed the DSC analysis. The DSC instrument conditions were: heating rate of $5{ }^{\circ} \mathrm{C} \mathrm{min}^{-1}$, heating from 30 to $100{ }^{\circ} \mathrm{C}$ under air atmosphere with flow of $50 \mathrm{~mL} \mathrm{~min}^{-1}$. The instrument was calibrated according to the manufacturer's specifications and verified with a Indium standard purity index of $99.999 \%$, m.p. $=156.6{ }^{\circ} \mathrm{C}, \Delta \mathrm{H}=28.71 \mathrm{~J} \mathrm{g.}{ }^{-1}$ (Lacerda et al., 2014; Andrade et al., 2014).

\section{Statistical analysis}

The results were expressed as mean \pm standard deviation and were analyzed using the Action Stat 3.3 software (Estatcamp, São Paulo, Brazil). To determine the behavior of the samples, unilateral analysis of variance - ANOVA was used. To determine the differences between the means, the Tukey test was used, with a 95\% confidence level ( $\mathrm{p}<0.05$ ) (Barretti, 2020).

\section{Results and discussion}

\section{Powder X-Ray diffractometry (PXRD)}

Potato starch presented pattern characteristics of BC type (B with characteristics of C) whereas the other three have a $\mathrm{C}$ pattern with $2 \theta$ at approximately $5.6^{\circ}, 15.3^{\circ} ; 17.3^{\circ}$ and $23.5^{\circ}$. Similar behavior were found in literature (Hung et al., 2016). It is possible to observe the decrease in peaks at $5.6^{\circ}$ according the HMT modified starches. In Figure 1, are depicted the X-ray diffractograms. They were used to analyze the main peaks and calculate the relative degree of crystallinity (\%) of each sample.

Native starch showed the highest relative crystallinity (27.2\%). Starch modified only with $\mathrm{HMT}+\mathrm{H}_{2} \mathrm{O}$ and starch modified with $\mathrm{HMT}+$ lactic acid shows similar crystallinity (25.6\%), the lowest relative crystallinity was observed for a sample modified with HMT + 
citric acid (19.9\%). These decreases can be explained due to a lamellar destabilization promoted by the treatment by HMT (Maior et al., 2020; Liu et al., 2019).

According to authors, the effect of heat treatment on starch crystallinity depends on the source of starch used and the conditions of moisture and heating used in the treatment. The decrease in crystallinity caused by the treatment of HMT combined with citric acid, which was $19.9 \%$, is much smaller when compared to the other values obtained, this can be explained by a possible decomposition of the crystalline structure of starch by citric acid, this phenomenon causes the substitution of citrate groups in the starch chains, forming a starch with limited mobility (Barretti et al., 2020; Andrade et al., 2014). Xia et al. (2016) also raises a reduction in relative crystallinity when studying sweet potato starches treated by HMT and citric acid.

On the other hand, other sudies showed an increasing in relative cristallinity values. It was observed that HMT, is able to transform the fraction of amorphous amylose to the crystalline state. It is possible that during the treatment the double helical chains help to form a crystalline matrix which presents a structure more ordered than an untreated starch (Zavareze et al., 2011)

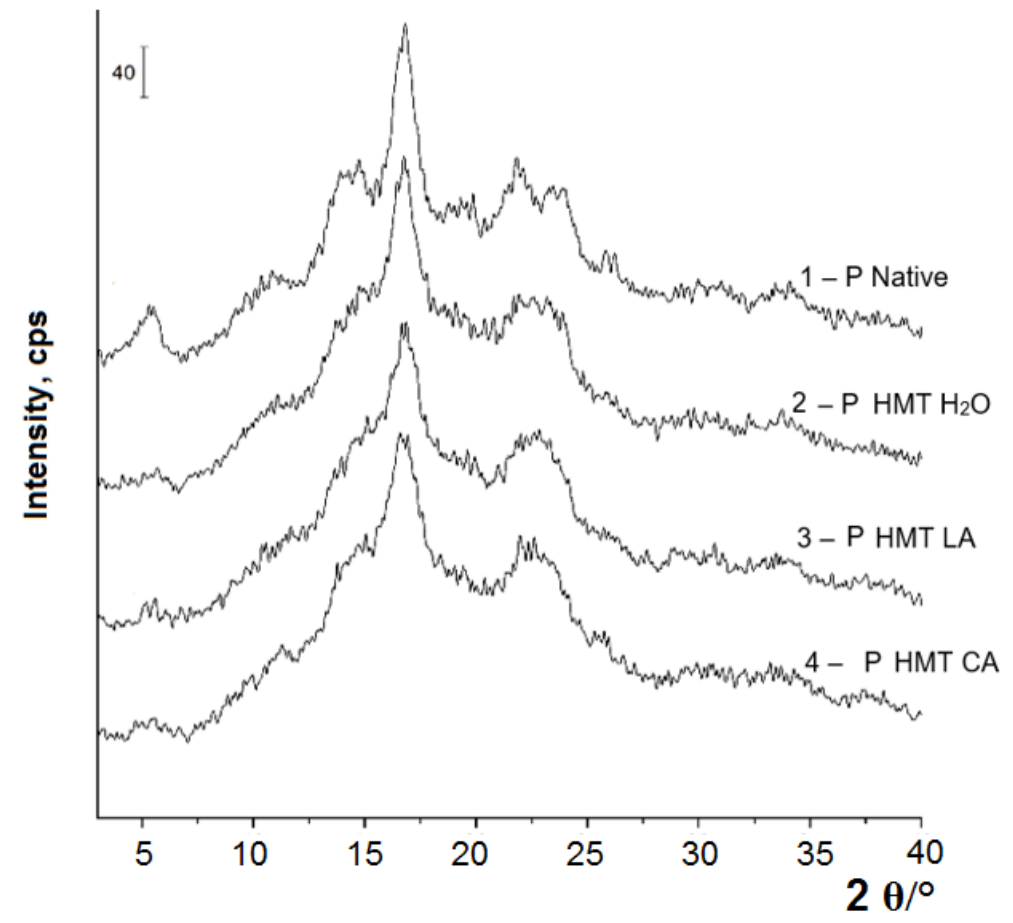

Figure 1. Diffractograms and relative crystallinity $(\mathrm{XC} \%)$ of native and modified potato starches

\section{Pasting properties (rapid viscoanalyser- RVA)}

Data relating to viscoamylographic analyzes of native and treated potato starch are provided in Table 1 and the viscosity profiles are illustrated in Figure 2. 
Table 1

Results obtained from the RVA curves of native and modified potato starches

\begin{tabular}{|c|c|c|c|c|c|c|}
\hline Samples & Treatments & $\begin{array}{c}\text { Pasting } \\
\text { temperature, } \\
\mathbf{}^{\mathbf{C}}\end{array}$ & $\begin{array}{c}\text { Viscosity } \\
\mathbf{p e a k}, \\
\mathbf{m P a} \cdot \mathbf{s}\end{array}$ & $\begin{array}{c}\text { Breakdown, } \\
\mathbf{m P a} \cdot \mathbf{s}\end{array}$ & $\begin{array}{c}\text { Setback, } \\
\mathbf{m P a} \cdot \mathbf{s}\end{array}$ & $\begin{array}{c}\text { Final } \\
\text { Viscosity, } \\
\mathbf{m P a} \cdot \mathbf{s}\end{array}$ \\
\hline Native & P Native & $65,50^{\mathrm{b}}$ & $7824,00^{\mathrm{a}}$ & $5868,5^{\mathrm{a}}$ & $847,00^{\mathrm{b}}$ & $2802,50^{\mathrm{b}}$ \\
& & $\pm 0,07$ & $\pm 1,41$ & $\pm 1,41$ & $\pm 2,12$ & $\pm 2,33$ \\
\hline $\mathrm{HMT}$ & P HMT $\mathrm{H}_{2} \mathrm{O}$ & $81,60^{\mathrm{a}}$ & $1732,00^{\mathrm{b}}$ & - & $1592,00^{\mathrm{a}}$ & $3329,00^{\mathrm{a}}$ \\
$+\mathrm{H}_{2} \mathrm{O}$ & & $\pm 0,57$ & $\pm 2,82$ & - & $\pm 1,41$ & $\pm 1,41$ \\
\hline HMT & P HMT LA & & $94,50^{\mathrm{c}}$ & $41,00^{\mathrm{b}}$ & $30,00^{\mathrm{c}}$ & $77,00^{\mathrm{c}}$ \\
+ LA & & - & $\pm 1,41$ & $\pm 0,05$ & $\pm 1,41$ & $\pm 2,12$ \\
\hline HMT & P HMT CA & - & $90,00^{\mathrm{d}}$ & $45,00^{\mathrm{c}}$ & $31,50^{\mathrm{c}}$ & $76,50^{\mathrm{c}}$ \\
+ CA & & - & $\pm 1,41$ & $\pm 0,02$ & $\pm 2,12$ & $\pm 0,71$ \\
\hline
\end{tabular}

$m P a \cdot s-$ millipascal-second, $s-$ second.Values presented as mean values \pm standard deviation. Values followed by the same letter in the same column are not significantly different by Tukey's test $(p<0.05)$

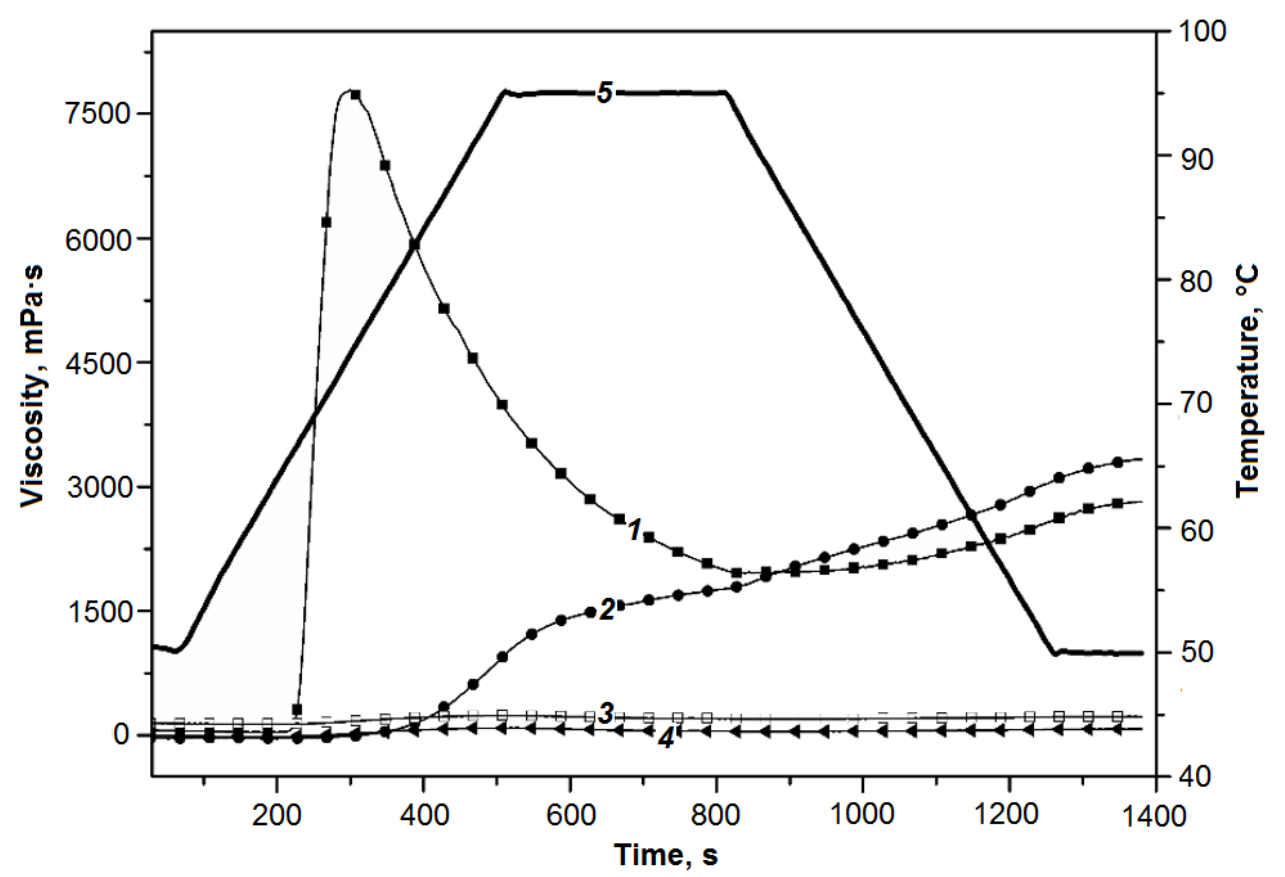

Figure 2. Pasting properties (RVA) curves of native and modified potato starches $(\mathrm{mPa} \cdot \mathrm{s})$

1 - P (Potato starch);

2 - $\mathrm{HMT}_{2} \mathrm{O}$ (Heat-moisture treatment with deionized water);

3 - HMT LA(heat-moisture treatment with lactic acid);

4 - HMT CA (Heat-moisture treatment with citric acid).

5 - temperature.

With the results obtained, it is possible to observe that the temperature for mass formation increases for $\mathrm{H}_{2} \mathrm{O}$ modified starch from 65.5 to $81{ }^{\circ} \mathrm{C}$, in relation to native starch. The transforms modified with the organic acids did not form masses, showing that the treatment of the starch by HMT with a gel formation. 
It is also possible to notice an increase in the final viscosity for $\mathrm{H}_{2} \mathrm{O}$ modified starch, which may be related to intra-granular curtains, making the starch more resistant to deformation, in addition to the reorganization of the starch chains. For how modified with citric and lactic acid, it hears a decrease in the final viscosity, suggesting the rupture of the granular structure (Barreti et al., 2020; Maior et al., 2020; Shaikh et al., 2019).

Viscosity reduction can occur by altering the amorphous fraction of the starch. In acidic conditions this amorphous fraction can be hydrolyzed and the starch starts to move more freely which can result in the closure of its chain and consequently starts to absorb less water which results in reduced viscosity (Maior et al., 2020).

In the sample of starch modified with citric and lactic acid, it is possible to observe that lower as lower final viscosities and peak viscosity, reduced breakage and less tendency to retrograde, this is due to the fact that they do not form paste. One of the major causes of texture deterioration in bakery products is retrogradation. Pastes with low viscosity values are desirable in these products since they have a lower tendency for this phenomenon to run (Hornung et al., 2015; Bet et al., 2020).

\section{Differential scanning calorimetry (DSC)}

In the Figure 3 it is possible to observe the DSC curves and in the Table 2 are the obtained values of $T_{o}, T_{p}, T_{c}$ as well as the gelatinization enthalpy $\Delta H_{\text {gel }}$ for the samples submitted to this analysis.

It can be observed that the gelatinization event occurred in native starch, with a single well-defined peak in the endothermic profile, which is due to the fact that they have low levels of lipids. In the other three samples, the absence or almost absence of the gelatinization curve shows that the changes made to the starch showed the expected decrease in the gelatinization characteristic. This is due to a rupture of the double helices or partial gelatinization of the amylose and amylopectin content during heating. It may also be due to the formation of new starch crystals that can present different thermal stability in the face of physical modification, leading to a high energy need for the dissociation of the crystals to occur and thus the gelatinization phenomenon to happen (Andrade et al., 2014).

Only two of the samples had the gelatinization event, being that of native starch and starch modified with $\mathrm{H}_{2} \mathrm{O}$. The native starch presented a higher $\Delta \mathrm{H}_{\text {gel }}$ value when compared to the $\mathrm{H}_{2} \mathrm{O}$ modified starch, showing that the $\mathrm{H}_{2} \mathrm{O}$ modification already reduces the gelatinization power of the starch. This change in the thermal characteristic of starch can be explained by the reduction of the destabilizing effect of the amorphous region in the fusion of the lens and by the dissociation of the double helices present in this region (Hung et al., 2017).

Regarding the values of $T_{o}, T_{p}$ and $T_{c}$ can see that they are similar between the two samples. In studies already carried out, the $\Delta \mathrm{H}_{\text {gel }}$ values of native potato starch revolve around similar values, as it is verified by (Andrade et al., 2014), where the value presented is $\Delta \mathrm{H}_{\text {gel }}$ (14.72 $\left.\mathrm{J} \mathrm{g}^{-1}\right)$ being very close to the value $\Delta \mathrm{H}_{\text {gel }}\left(14.5 \mathrm{~J} \mathrm{~g}^{-1}\right)$ found in the present study. The starches modified with organic acids do not show gelatinization (It is observed an enlargenment between $T_{o}$ and $T_{c}$ with drastic reduction of viscosity and impossible to stablish values). This behavior can be explained by the occurrence of an esterification reaction caused by these acids (Barreti et al., 2020). 


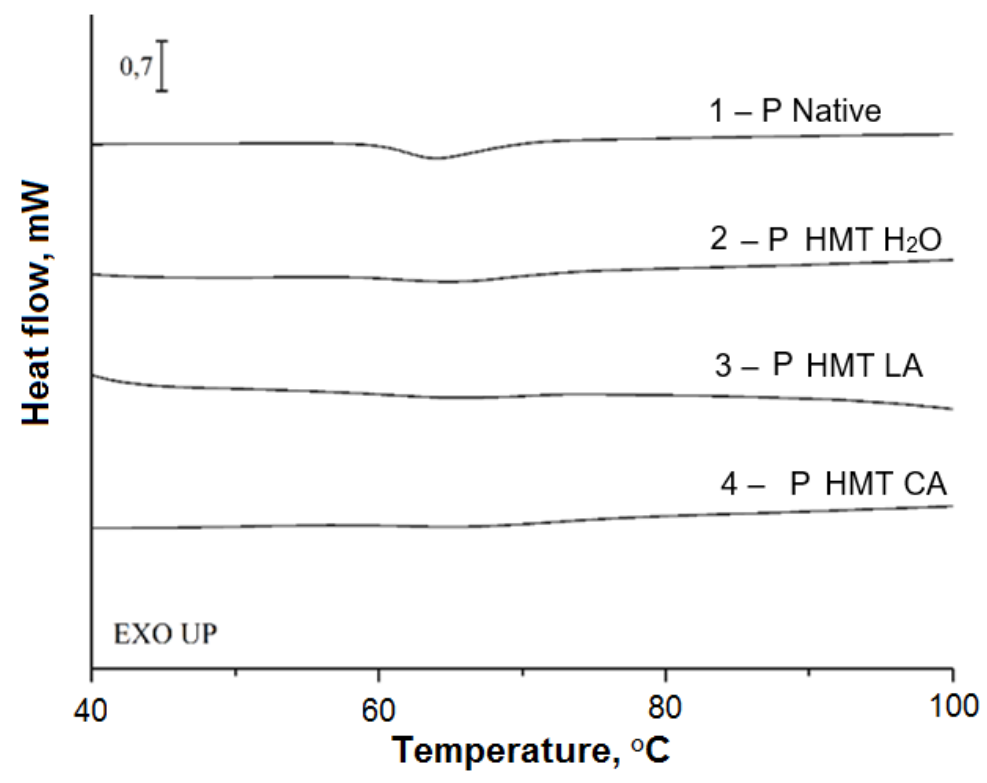

Figure 3. DSC curves of native and modified potato starches 1 - P (Potato starch);

2 - $\mathrm{HMT} \mathrm{H}_{2} \mathrm{O}$ (Heat-moisture treatment with deionized water); 3 - HMT LA(heat-moisture treatment with lactic acid); 4 - HMT CA (Heat-moisture treatment with citric acid).

Table 2. Results of DSC curves for native and modified potato starches

\begin{tabular}{|c|c|c|c|c|}
\hline Samples & $\begin{array}{c}\mathbf{T}_{\mathbf{o}}, \\
{ }^{\circ} \mathbf{C}\end{array}$ & $\begin{array}{c}\mathbf{T} \mathbf{T}, \\
{ }^{\circ} \mathbf{C}\end{array}$ & $\begin{array}{c}\mathbf{T}_{\mathbf{c}}, \\
{ }^{\circ} \mathbf{C}\end{array}$ & $\begin{array}{c}\Delta \mathbf{H}_{\text {gel }} \\
\mathbf{J ~ g}^{-1}\end{array}$ \\
\hline Native & $59,8^{\mathrm{a}} \pm 0,06$ & $64,0^{\mathrm{a}} \pm 0,01$ & $70,6^{\mathrm{a}} \pm 0,09$ & $14,5^{\mathrm{a}} \pm 0,08$ \\
\hline HMT $+\mathrm{H}_{2} \mathrm{O}$ & $58,5^{\mathrm{b}} \pm 0,16$ & $64,9^{\mathrm{a}} \pm 0,01$ & $72,9^{\mathrm{b}} \pm 0,14$ & $9,9^{\mathrm{b}} \pm 0,29$ \\
\hline HMT + LA & - & - & - & - \\
\hline HMT + CA & - & - & - & - \\
\hline
\end{tabular}

$T_{\mathrm{o}}$ - onset temperature, $T_{\mathrm{p}}$ - peak temperature, $T_{\mathrm{c}}-$ conclusion temperature, $\Delta \mathrm{H}_{\text {gel }}-$ gelatinisation enthalpy. Values presented as mean values \pm standard deviation. Values followed by the same letter in the same column are not significantly different by Tukey's test $(p<0.05)$

According to (Maior et al., 2020) corn starch showed a higher $\Delta \mathrm{H}_{\text {gel }}$ when compared to starches that underwent HMT modification, and that the modification combined with organic acids promoted an even greater reduction in $\Delta \mathrm{H}_{\text {gel }}$. Regarding the values of $T_{o}, T_{p}, T_{c}$, the work reports that after the modification the values obtained were higher, being in accordance with the shown results. Other studies have also shown similarities in the behavior of starches when subjected to HMT combined with organic acids (Barreti et al., 2020; Hung et al., 2016; Hung et al., 2017; Xia et al., 2016). 


\title{
Conclusions
}

Starch modified by HMT presents with appropriate and different characteristics comparing to the native form. It was possible to observe changes on gelatinization, cristallinity, and pasting properties. The treatment of potato starch using $\mathrm{HMT}+\mathrm{H}_{2} \mathrm{O}$, showed some properties alterations, however the modifications were more evident for the treatments with HMT + organic acids, which presented important results and altered technological properties such as the viscosity profile of the samples. The DSC and RVA analysis showed that the characteristic thickener of the starch can be lost after the modifications combined with the organic acids. PXRD analysis showed a decrease in the relative crystallinity after the modifications. The heat-moisture treatment with lactic and citric acids can be considered safe and ecologically correct treatments with organic acids of food grade. Processed modified samples proved to be suitable for application in foods that require low viscosity, such baby food and soups for example. On the other hand, treated granular starch can be an interesting alternative to be used as ingredient for baked products like cookies. It is possible that they have altered the digestibility of this polymer in the human body.

\begin{abstract}
Acknowledgements. The authors would like to thank CNPq - Brazilian Council for Scientific and Technological Development (Proc. $\mathrm{n}^{\mathrm{o}} .310534 / 2020-8$ and 308515/2018-8) and CAPES - Brazilian Coordination for the improvement of Personell in High Level.
\end{abstract}

\section{References}

Andrade M.M.P., De Oliveira C.S., Colman T.A.D., Da Costa F.J.O.G., Schnitzler E. (2014), Effects of heat-moisture treatment on organic cassava starch: Thermal, rheological and structural study, Journal of Thermal Analysis and Calorimetry, 115, pp. 2115-22.

Barretti B.R.V., Almeida V.S., Ito V.C., Silva B.M., Carvalho Filho M.A.S., Sydney E.B., Demiate I.M., Lacerda L.G. (2020), Combination of organic acids and heat-moisture treatment on the normal and waxy corn starch: thermal, structural, pasting properties, and digestibility investigation, Food Science and Technology, 2061, pp. 1-7.

Bet C.D., Bisinella R Z B., Colman T.A.D., Lacerda L.G., Schnitzler E. (2020), Morphology of starch from Araucaria angustifolia seeds treated by HMT and studied by SEM, AFM and XRD, Ukrainian Food Journal 9, pp 769-779.

Colussi R., Kringel D., Kaur L., da Rosa Zavareze E., Dias A.R.G., Singh J. (2020), Dual modification of potato starch: Effects of heat-moisture and high pressure treatments on starch structure and functionalities, Food Chemistry, 318.

dos Santos T.P.R., Leonel M., Garcia É.L., do Carmo E.L., Franco C.M.L. (2016), Crystallinity, thermal and pasting properties of starches from different potato cultivars grown in Brazil. International Journal of Biological Macromolecules, 82, pp.144-9.

Hornung P.S., Granza A.G., de Oliveira C..S, Lazzarotto M., Schnitzler E. (2015), Study of the Effects of Ultraviolet Light and Sodium Hypochlorite Solutions on Properties of Cassava Starch Granules, Food Biophysics, 10, pp. 368-74.

Hung P., Thi N., Huong M., Thi N., Phi L. (2017), Physicochemical characteristics and in vitro digestibility of potato and cassava starches under organic acid and heat-moisture treatments, International Journal of Biological Macromolecules, 95, pp. 299-305.

Hung P.V., Chau H.T., Phi N.T.L. (2016), In vitro digestibility and in vivo glucose response of native and physically modified rice starches varying amylose contents, Food Chemistry, 191, pp. 74-80. 
Hung P.V., Vien N.L., Lan P.N.T. (2016), Resistant starch improvement of rice starches under a combination of acid and heat-moisture treatments, Food Chemistry, 191, pp. 67-73.

Jiranuntakul W., Puttanlek C., Rungsardthong V., Puncha-Arnon S., Uttapap D. (2011), Microstructural and physicochemical properties of heat-moisture treated waxy and normal starches, Journal of Food Engineering, 104, pp. 246-58.

Kubiaki F.T., Figueroa A.M., de Oliveira C.S., Demiate I.M., Schnitzler E., Lacerda L.G. (2018), Effect of acid-alcoholic treatment on the thermal, structural and pasting characteristics of European chestnut (Castanea sativa, Mill) starch, Journal of Thermal Analysis and Calorimetry, 131, pp. 587-94.

Lacerda L.G., Colman T.A.D., Bauab T., Carvalho Filho M.A.S., Demiate I.M., De Vasconcelos E.C., Schnitzler E. (2014), Thermal, structural and rheological properties of starch from avocado seeds (Persea americana, Miller) modified with standard sodium hypochlorite solutions, Journal of Thermal Analysis and Calorimetry, 115, pp. 1893-9.

Leonel M., do Carmo E.L., Leonel S., Franco C.M.L., Campanha R.B. (2011), Starch extraction and characterization of different genotypes of banana, Revista Brasileira de Fruticultura, 33.

Liu K., Zhang B., Chen L., Li X., Zheng B. (2019), Hierarchical structure and physicochemical properties of highland barley starch following heat moisture treatment, Food Chemistry, 271, 102-8.

Maior L.O., Almeida V.S., Barretti B.R.V., Ito V.C., Beninca C., Demiate I.M., Schnitzler E., Carvalho Filho M.A.S., Lacerda L.G. (2020), Combination of organic acid and heat-moisture treatment: impact on the thermal, structural, pasting properties and digestibility of maize starch. Journal of Thermal Analysis and Calorimetry, 1, pp. 1-8.

Nara S., Komiya T. (1983), Studies on the Relationship Between Watersatured State and Crystallinity by the Diffraction Method for Moistened Potato Starch. Starch-Stärke, 35 pp. $407-$ 10 .

Noda T., Tsuda S., Mori M., Takigawa S., Matsuura-Endo C., Kim S.J., Hashimoto N., Yamauchi H. (2006), Determination of the phosphorus content in potato starch using an energydispersive X-ray fluorescence method, Food Chemistry, 95, pp. 632-7.

Pereira K.D. (2007), Resistant starch, the latest generation of energy control and healthy digestion, Ciencia e Tecnologia de Alimentos, 27, pp. 88-92.

Schafranski K., Ito V.C., Lacerda L.G. (2021), Impacts and potential applications: A review of the modification of starches by heat-moisture treatment (HMT), Food Hydrocolloids, 117, pp. 106690.

Shaikh F., Ali T.M., Mustafa G., Hasnain A. (2019), Comparative study on effects of citric and lactic acid treatment on morphological, functional, resistant starch fraction and glycemic index of corn and sorghum starches, International Journal of Biological Macromolecules, 135, pp. 314-27.

Shin S.I., Lee C.J., Kim D.I., Lee H.A., Cheong J.J., Chung K.M.,Baik M.Y., Park C.S., Kim C.H., Moon T.W. (2007), Formation, characterization, and glucose response in mice to rice starch with low digestibility produced by citric acid treatment, Journal of Cereal Science, 45, pp. 24-33.

Xia H., Li Y., Gao Q. (2016), Preparation and properties of RS4 citrate sweet potato starch by heat-moisture treatment, Food Hydrocolloids, 55, pp.172-8.

Xu M., Saleh A.S.M., Gong B., Li B., Jing L., Gou M., Jiang H., Li W. (2018), The effect of repeated versus continuous annealing on structural, physicochemical, and digestive properties of potato starch, Food Research International, 111, pp. 324-33.

Yassaroh Y., Woortman A.J.J., Loos K. (2019), A new way to improve physicochemical properties of potato starch, Carbohydrate Polymers, 204, pp. 1-8.

Zaman S.A., Sarbini S.R. (2016), The potential of resistant starch as a prebiotic, Critical Reviews in Biotechnology, 36, pp. 578-84.

Zavareze E.R., Dias A.R.G. (2011), Impact of heat-moisture treatment and annealing in starches: A review, Carbohydrate Polymers, 83, pp. 317-328. 